Recibido 21/06/2018 --- Aceptado: 12/09/2018 --- Publicado: 15/03/2019

\title{
RELACIÓN ENTRE LOS BENEFICIOS Y LA OBTENCIÓN DE ENGAGEMENT DE LOS USUARIOS EN LA COMUNICACIÓN DE LAS REDES SOCIALES DEL SECTOR HOTELERO
}

\section{RELATIONSHIP BETWEEN THE BENEFITS AND THE OBTAINING OF ENGAGEMENT OF THE USERS IN THE COMMUNICATION OF THE SOCIAL NETWORKS OF THE HOTEL SECTOR}

Miguel Ángel Sánchez Jiménez: Universidad de Cádiz. España. miguelangel.sanchez@uca.es

María Teresa Fernández Allés: Universidad de Cádiz. España. teresa.alles@uca.es

Juan José Mier-Terán Franco: Universidad de Cádiz. España. Juanjose.mier-teran@uca.es

\section{RESUMEN}

Con el creciente poder de los consumidores online y la mayor penetración de internet en todo el mundo, las comunidades online de marcas han formado una importante plataforma de comunicación entre las empresas y los consumidores. Estos medios sociales han ofrecido al consumidor la oportunidad de compartir opiniones, recomendaciones y experiencias a través de comentarios, imágenes o videos con otros usuarios, que pueden ser leídos y comentados entre ellos. De esta manera, en esta investigación se pretende analizar si los beneficios percibidos están relacionados con la obtención de compromiso o engagement por parte de los usuarios en las redes sociales de los hoteles. Para ello se ha realizado un análisis cuantitativo mediante un cuestionario dirigido a los seguidores de las redes sociales de hoteles o cadena de hoteles. Así, se han propuesto 4 hipótesis para analizar si los 4 beneficios identificados (beneficio funcional, socio-psicológico, hedónico y monetario) están directamente relacionados con las 4 variables identificadas del engagement (participación del usuario, confianza, compromiso afectivo y lealtad) pudiendo contrastar así si los beneficios percibidos por los usuarios en dichas redes sociales provocado un aumento en las 4 variables del engagement. Los resultados especifican una vinculación positiva de los beneficios sociopsicológicos y hedónicos percibidos por el usuario con un mayor engagement, sin embargo, hay más discrepancia en los

${ }^{1}$ Miguel Ángel Sánchez Jiménez: Profesor doctor en el Departamento de Marketing y Comunicación de la Universidad de Cádiz.

miquelangel.sanchez@uca.es 
Sánchez Jiménez, M. A.; Fernández Allés, M. T. y Mier-Terán Franco, J. J. Relación entre los beneficios y la obtención de engagement de los usuarios en la comunicación de las redes sociales del sector hotelero

beneficios funcionales $y$, sobre todo, destacar la escasa trascendencia del beneficio monetario para conseguir un mayor compromiso con los usuarios.

PALABRAS CLAVE: redes sociales - comunicación - sector hotelero - usuarios, beneficios - engagement - cuestionario.

\section{ABSTRACT}

With the growing power of online consumers and the increased penetration of the internet around the world, online brand communities have formed an important communication platform between companies and consumers. These social media have offered consumers the opportunity to share opinions, recommendations and experiences through comments, images or videos with other users, which can be read and commented among them. In this way, this research intends to analyze if the perceived benefits are related to the obtaining of commitment or engagement on the part of the users in the social networks of the hotels. For this, a quantitative analysis has been carried out by means of a questionnaire addressed to the followers of the social networks of hotels or hotel chains. Thus, 4 hypotheses have been proposed to analyze whether the 4 identified benefits (functional, sociopsychological, hedonic and monetary benefits) are directly related to the 4 variables identified in the engagement (user participation, trust, affective commitment and loyalty), being able to contrast so if the benefits perceived by users in these social networks caused an increase in the 4 variables of engagement. The results specify a positive link of the socio-psychological and hedonic benefits perceived by the user with a greater engagement, however, there is more discrepancy in the functional benefits and, above all, to highlight the scarce transcendence of the monetary benefit to achieve a greater commitment with the users.

KEY WORDS: social networks - communication - hotel sector - users - benefits, engagement - questionnaire.

\section{RELAÇÃO ENTRE OS BENEFÍCIOS E A OBTENÇÃO DE ENGAGEMENT DOS USUÁRIOS NA COMUNICAÇÃO DAS REDES SOCIAIS DO SETOR HOTELEIRO}

\section{RESUME}

Com o crescente poder dos consumidores online e a maior penetração de internet em todo o mundo, as comunidades online de marcas formaram uma importante plataforma de comunicação entre as empresas e os consumidores. Esses meios sociais ofereceram ao consumidor a oportunidade de compartir opiniões, recomendações e experiências através de comentários, imagens ou vídeos com outros usuários, que podem ser lidos e comentados entre si. Desta maneira, nesta investigação pretende-se analisar se os benefícios percebidos estão relacionados com a obtenção de compromisso ou engagement por parte dos usuários nas redes sociais 
Sánchez Jiménez, M. A.; Fernández Allés, M. T. y Mier-Terán Franco, J. J. Relación entre los beneficios y la obtención de engagement de los usuarios en la comunicación de las redes sociales del sector hotelero

dos hotéis. Para isso foi realizado uma analises quantitativa mediante um questionário dirigido aos seguidores das redes sociais de hotéis ou cadeia de hotéis. Assim, propuseram 4 hipóteses para analisar se os 4 benefícios identificados (benefício funcional, sócio psicológico, hedônico e monetário) estão diretamente relacionados com as 4 variáveis identificadas do engagement (participação do usuário, confiança, compromisso afetivo e lealdade) podendo contrastar assim se os benefícios percebidos pelos usuários em tais redes sociais provocaram um aumento nas 4 variáveis do engagement. Os resultados especificam uma vinculação positiva dos benefícios sócio psicológicos e hedônicos percebidos pelo usuário com um maior engagement, embora haver mais discrepância nos benefícios funcionais e, sobretudo, destacar a escassa transcendência do benefício monetário para conseguir um maior compromisso com os usuários.

PALAVRAS CHAVE: redes sociais - comunicação - setor hoteleiro - usuários benefícios, engagement - questionário.

\section{Cómo citar el artículo:}

Sánchez Jiménez, M. A.; Fernández Allés, M. T. y Mier-Terán Franco, J. J. (2019). Relación entre los beneficios y la obtención de engagement de los usuarios en la comunicación de las redes sociales del sector hotelero. [Relationship between the benefits and the obtaining of engagement of the users in the communication of the social networks of the hotel sector]. Revista de Comunicación de la SEECI, 48, 125-148. doi: http://doi.org/10.15198/seeci.2019.48.125-148 Recuperado de http://www.seeci.net/revista/index.php/seeci/article/view/548

\section{INTRODUCCIÓN}

Los medios sociales han ofrecido al consumidor la oportunidad de compartir opiniones, recomendaciones y experiencias a través de comentarios, imágenes 0 videos con otros usuarios, que pueden ser leídos y comentados entre ellos. Esto ha llevado a que muchas empresas turísticas se hayan lanzado a probarlo y tengan la oportunidad tanto de analizar la información como de comunicarse con el consumidor, dando paso a la promoción de ventas con el objetivo de crear un mensaje centrado en el cliente (Martínez et al., 2013). Así, Los medios sociales están cambiando la forma en que el turista consume y contribuye a la creación de información, suponiendo una alteración radical en la manera en que la información se difunde (Potgieter y Naidoo, 2017; Rosenthal y Brito, 2017). Además, las experiencias relacionadas con el turismo no pueden ser valoradas hasta que se consumen, puesto que son intangibles, de ahí que los medios sociales, con las recomendaciones personales de los usuarios y de personas cercanas, sean muy influyentes a la hora de tomar una decisión sobre la elección turística (Buhalis, 1998; Litvin, et al., 2008).

Las características propias del sector turístico, en general, y del sector hotelero, en particular, hacen que sea mayor su repercusión en los medios sociales. El sector turístico, como cualquier sector servicios, vende productos intangibles que se 
Sánchez Jiménez, M. A.; Fernández Allés, M. T. y Mier-Terán Franco, J. J. Relación entre los beneficios y la obtención de engagement de los usuarios en la comunicación de las redes sociales del sector hotelero

caracterizan por ser inseparables, perecederos y heterogéneos (Sirakaya y Woodside, 2005), de ahí que el proceso de comunicación sea un elemento vital en la oferta (Sigala, 2007). Por esta razón, el uso de los medios sociales ha permitido nuevas posibilidades de comunicación y ha proporcionado un impacto sustancial en la demanda y la oferta turística (Martínez et al., 2013).

Sahín y Sengün (2015) especifican que los comentarios en medios sociales son importantes debido a la gran proporción de usuarios que utilizan internet para decidir su estancia. En particular, los usuarios que quieren tener información sobre los destinos turísticos y los establecimientos hoteleros, son influenciados en gran medida por los comentarios en las redes sociales, por lo tanto, el reconocimiento de marca y la confianza en el establecimiento depende de estos comentarios. Esto deriva en que la influencia de los medios sociales en el ámbito hotelero sea alta, permitiendo a los establecimientos turísticos ponerse en contacto con los usuarios y que sus acciones lleguen a una gran cantidad de personas en un corto periodo de tiempo y de una manera eficiente (Bambauer-Sachse y Mangold, 2011).

Esto es especialmente relevante para las empresas hoteleras ya que dependen en gran medida de la experiencia del cliente, por lo que deben preocuparse más en mejorar engagement o compromiso (Dowling, 2001). En este contexto, el engagement del consumidor ha surgido como un concepto dinámico e interactivo a través del que poder entender la construcción de relaciones fuertes, duraderas y fieles con los clientes. El concepto de engagement del consumidor supera la perspectiva individualista del marketing tradicional para proporcionar a los vendedores un proceso global que abarca todas las actividades, basándose para ello en la marca y en su motivación sobre los clientes, dando lugar a lazos emocionales y cercanos entre el cliente y la empresa (Naumann y Lay-Hwa Bowden, 2015).

De acuerdo con Cruz y Mendelsohn (2010), los miembros de las comunidades online de marcas están más comprometidos y son más propensos a comprar y recomendar la marca a los demás, en comparación con los no miembros. En el mismo sentido, Becerra y Badrinarayanan (2013) determinan que los miembros de la comunidad online de una marca contribuyen en mayor medida a la difusión de la información sobre dicha marca. Por lo tanto, se considera de gran interés comprender como los usuarios intervienen en estas comunidades online de marcas y conocer cuáles son los beneficios que estos perciben. En este sentido, resulta esencial analizar el engagement o compromiso del consumidor. Conocer el engagement del consumidor hacia un destino turístico aporta un valor añadido al mismo, ya que se basa en el incremento de la interactividad y en la mejora de las relaciones con el consumidor (Vivek et al., 2014).

De esta manera, el objetivo de este estudio es conocer analizar si los beneficios percibidos están relacionados con la obtención de compromiso o engagement por parte de los usuarios en las redes sociales de los hoteles. Para ello se ha realizado un análisis cuantitativo mediante un cuestionario dirigido a los seguidores de las redes sociales de hoteles o cadena de hoteles. Así, se han propuesto 4 hipótesis para analizar si los 4 beneficios identificados (beneficio funcional, socio-psicológico, 
Sánchez Jiménez, M. A.; Fernández Allés, M. T. y Mier-Terán Franco, J. J. Relación entre los beneficios y la obtención de engagement de los usuarios en la comunicación de las redes sociales del sector hotelero

hedónico y monetario) están directamente relacionados con las 4 variables identificadas del engagement (participación del usuario, confianza, compromiso afectivo y lealtad) pudiendo contrastar así si los beneficios percibidos por los usuarios en dichas redes sociales provocado un aumento en las 4 variables del engagement.

\section{HIPÓTESIS DE INVESTIGACIÓN}

En este epígrafe se plantean las hipótesis de la investigación que suponen hechos observables que deben de ser contrastados empíricamente (Tercero, 1999).

H1: un mayor beneficio funcional, socio-psicológico, hedónico y monetario se asocia con una mayor participación en la comunidad online del hotel.

H2: un mayor beneficio funcional, socio-psicológico, hedónico y monetario se asocia con una mayor confianza hacia el hotel.

H3: un mayor beneficio funcional, socio-psicológico, hedónico y monetario se asocia con un mayor compromiso afectivo hacia el hotel.

H4: un mayor beneficio funcional, socio-psicológico, hedónico y monetario se asocia con una mayor lealtad hacia el hotel.

\section{MARCO TEÓRICO}

A continuación, se especifica los estudios más relevantes acerca de los principales conceptos que se han tenido en cuenta en esta investigación: los beneficios percibidos por los usuarios y las variables del engagement identificadas.

\subsection{Beneficios funcionales, psicológicos, sociales, hedónicos y económicos de las comunidades online}

Varias teorías sociales han explicado por qué las personas utilizan las redes sociales (en este caso, redes sociales de cadenas hoteleras): para obtener información; hacer compras; para comunicarse e interactuar con otros; para divertirse y disfrutar de la experiencia; para entablar nuevas relaciones; y para expresar sus opiniones y su identidad. De los estudios realizados sobre las investigaciones de Kang (2011), Leung et al. (2015), Vivek et al. (2012) y Naumann y Bowden (2015), y observadas las opiniones de expertos en redes sociales, se han identificado las principales variables objeto de estudio y propuesto varios modelos para contrastar las hipótesis básicas que están relacionadas con el beneficio percibido por el usuario y el compromiso del consumidor.

En diversas investigaciones se ha mostrado interés por conocer los factores que motivan la participación y el compromiso de los usuarios de las redes sociales. Wang y Fesenmaier (2004) propusieron un modelo basado en las necesidades o beneficios que los usuarios buscan satisfacer en las comunidades virtuales. Según estos autores, la participación y el compromiso están impulsados por cuatro tipos de 
Sánchez Jiménez, M. A.; Fernández Allés, M. T. y Mier-Terán Franco, J. J. Relación entre los beneficios y la obtención de engagement de los usuarios en la comunicación de las redes

necesidades 0 beneficios: funcionales, sociales, psicológicos y hedónicos. Argumentan que para que las comunidades virtuales sean viables deben atender a una variedad de necesidades o beneficios de los miembros. En el estudio de Dholakia et al. (2004) identificaron factores más específicos que motivan a los individuos a participar en comunidades virtuales basados en la acción intencional. Madupu (2006) afirmó que los motivos de Dholakia et al. (2004) pueden conciliarse y relacionarse con las categorías de beneficios propuestas por Wang y Fesenmaier (2004), proponiendo así un conjunto de antecedentes para la participación y compromiso en las comunidades virtuales (Tabla 1 ).

Tabla 1. Beneficios de la participación y el compromiso en las comunidades virtuales de una marca.

\begin{tabular}{|c|c|}
\hline Necesidades o beneficios & Factores motivacionales \\
\hline \multirow{2}{*}{ Funcionales } & Información/Utilidad \\
& Eficiencia \\
& Conveniencia \\
\hline \multirow{2}{*}{ Sociales } & Confianza \\
& Comunicación \\
& Relación \\
Psicológicas & Implicación \\
\hline \multirow{2}{*}{ Hedónicas } & Pertenencia \\
& Afiliación \\
& Identificación \\
\hline & Entretenimiento \\
& Diversión \\
& Placer \\
\hline
\end{tabular}

Fuente: Elaboración propia a partir de los trabajos de Madupu (2006), Dholakia et al. (2004) y Wang y Fesenmaier (2004).

Además de estas cuatro variables, se tendrán en cuenta los beneficios monetarios que han sido valorados por otros estudios como el Gwinner et al. (1998) y Lee et al. (2008). Esta dimensión se relaciona con descuentos o eventos de la empresa ofrecidos a la comunidad "obtener descuentos o tratos especiales que la mayoría de consumidores no tienen", "obtener mejores precios que la mayoría de consumidores" y "recibir cupones gratis para una estancia en el hotel o de comida por hacerse miembro de la página de Facebook".

El beneficio funcional se entiende como la facilidad en la obtención de información relevante para los objetivos del consumidor sin tener limitaciones temporales 0 geográficas (De Vries y Carlson, 2014), así como la facilidad para realizar las transacciones pertinentes. Uno de los beneficios más visibles de las redes sociales es que la información se encuentre públicamente disponible y sea de fácil acceso (Hays y Buhalis, 2013). Algunos estudios (Hiltz y Wellman, 1997) afirman que el conocimiento y la información son un activo valioso para las comunidades. Si los miembros pueden conseguir beneficios funcionales, como adquirir información rápidamente, es más probable que visiten la comunidad online (Al-Msallan y Alhaddad, 2016). En cualquier caso, la relación que haya entre los beneficios 
Sánchez Jiménez, M. A.; Fernández Allés, M. T. y Mier-Terán Franco, J. J. Relación entre los beneficios y la obtención de engagement de los usuarios en la comunicación de las redes sociales del sector hotelero

funcionales y el compromiso en la comunidad variará en función de lo que los usuarios busquen obtener de la comunidad (por ejemplo, si prefieren entretenimiento o adquirir información). En el estudio de Al-Msallan y Alhaddad (2016), los miembros de la página de Facebook de los hoteles buscaban una comunicación con el resto de miembros de forma eficiente, así como compartir información sobre sus experiencias con las marcas de hoteles. Para cumplir estos deseos, los miembros visitaban las redes sociales de forma frecuente. Según este estudio, los beneficios funcionales tienen una influencia directa con el incremento de la participación de los miembros en las páginas de Facebook.

Los beneficios sociales percibidos están relacionados con el apoyo emocional, la amistad o la comunicación con otros miembros (Chen, 2013). Algunos estudios han revelado que las comunidades virtuales son lugares donde la gente busca un apoyo emocional y un sentido de permanencia Furlong, 1989; Hiltz y Wellman, 1997). Cuatro factores del beneficio social: pertenencia, influencia y relación, integración y satisfacción de una necesidad, junto con la conexión con las emociones compartidas, fueron utilizados en el estudio de Kim et al. (2004), comprobando que los tres primeros tenían un efecto directo en la intención de compra. Este tipo de beneficios deriva de los sentimientos de conexión con los miembros de la comunidad e incluyen el sentimiento de afiliación con los otros individuos (Bressler y Grantham, 2000). Como miembro se obtiene información sobre la comunidad y se empieza a percibir un sentimiento de pertenencia a dicho grupo, lo que deriva en un símbolo de identificación (Wang y Fesenmaier, 2004).

La relación significativa entre los beneficios psicológicos y la participación en las comunidades se muestra en los estudios de Ahuja y Galvin (2003), Langerak et al. (2003) y Al-Msallan y Alhaddad (2016). Así, se indica que los beneficios psicológicos influyen de forma significativa en las actitudes de los miembros hacia la comunidad online, también encontró una relación positiva entre el sentido de pertenencia a una comunidad y la lealtad hacia la misma. Estos estudios han probado también la relación positiva que se da entre los beneficios psicológicos y el compromiso en una comunidad online. Los consumidores quizás incrementen su nivel de participación para expresar sus preferencias, lo que le une emocionalmente al resto de miembros de la comunidad.

En los estudios de Kang (2011) y Song y Yoo (2016) establecen que los beneficios sociales y psicológicos tienen una alta correlación entre ellos, por lo tanto, en ambos estudios, se combinaron ambas variables en una rebautizada como "beneficio sociopsicológico". Debido a que se ha demostrado alta correlación entre estas dos variables, en este estudio también se han combinado dando como resultado la variable "beneficio socio-psicológico" la cual ha sido objeto de estudio.

Incluye estados emocionales positivos como la diversión y el disfrute de la experiencia cuando se participa en actividades de la comunidad (Wang y Fesenmaier, 2004). En las comunidades online, los miembros pueden engancharse a actividades que les ocasionan emociones positivas como la felicidad, la excitación o el entusiasmo (Dholakia (2004). Algunas comunidades online permiten participar en 
Sánchez Jiménez, M. A.; Fernández Allés, M. T. y Mier-Terán Franco, J. J. Relación entre los beneficios y la obtención de engagement de los usuarios en la comunicación de las redes sociales del sector hotelero

juegos, concursos o realizar encuestas, lo que suponen diversión y entretenimiento (Wang y Fesenmaier, 2004).

Los consumidores esperan obtener una ventaja económica de una relación, entendida esta como un ahorro monetario (Gwinner et al., 1998). Por ejemplo, obtener descuentos es una de las razones por las que los individuos se relacionan con una marca (Harris et al., 2003; Gwinner et al. (1998) mostraron la importancia de los beneficios monetarios cuando se pretende desarrollar una relación con una empresa de servicios. Como califican Al-Msallan y Alhaddad (2016), los beneficios monetarios necesitan ser más investigados para determinar si pueden estimular a los consumidores potenciales a unirse a las páginas de Facebook de los hoteles. En concreto, en su investigación muestran que no influyen en el nivel de compromiso de las comunidades online.

\subsection{Variables del engagement: Participación del consumidor, confianza hacia la marca, la lealtad hacia la marca, y el compromiso afectivo}

El engagement o compromiso del consumidor es considerado uno de los principales atractivos del uso del social media marketing, por lo tanto, se estima de interés relacionar estas variables de los tipos de beneficios con aquellas que más se identifican e influyen en el compromiso del consumidor. Tras una revisión de la literatura se han tenido en cuenta las siguientes variables que afectan al compromiso del consumidor hacia la marca: la participación del consumidor en las redes sociales, la confianza hacia la marca, el compromiso afectivo y la lealtad hacia la marca.

Los miembros pasivos raramente se involucran en las actividades de la comunidad. El número de estos usuarios generan tráfico y aumentan las visitas del sitio web pero no garantizan a la comunidad el éxito ya que no contribuyen a las actividades comunitarias. Por el contrario, este tipo de miembros tienden a perseguir sus propias metas (Al-Msallan y Alhaddad, 2016). Wang y Fesenmaier (2004) demostraron empíricamente que las necesidades hedónicas y sociales están relacionadas positivamente con la participación mientras que los beneficios funcionales tienen un efecto negativo. Según estos autores, las necesidades psicológicas no tienen relación con la participación. En el estudio realizado por Al-Msallan y Alhaddad (2016) se mostró una relación positiva entre los beneficios funcionales, sociales, psicológicos y hedónicos con la participación en la comunidad. Por el contrario, los beneficios económicos no tienen una relación significativa con la participación.

Brodie et al. (2011), por su parte, define la confianza en función de las comunidades online, entendiendo como confianza la de los miembros de una comunidad, haciendo que el grupo sea eficiente y resuelva los problemas que puedan surgirle a alguno de ellos. Uno de los objetivos del marketing es ganar la confianza de los consumidores en la marca, ya que la confianza es importante durante el proceso de compra (Powers et al., 2012). Esta importancia repercute en las actitudes y comportamientos que los consumidores adoptan frente a una marca (Gefen et al., 2003). Las comunidades online se están convirtiendo en fuentes de información desde que los viajeros tienen más confianza en las opiniones del resto 
Sánchez Jiménez, M. A.; Fernández Allés, M. T. y Mier-Terán Franco, J. J. Relación entre los beneficios y la obtención de engagement de los usuarios en la comunicación de las redes sociales del sector hotelero

de los miembros que en las organizaciones de marketing (Gretzel et al., 2008). Por lo tanto, a priori, los beneficios sociales y funcionales influirán en la confianza que tenga un consumidor hacia una determinada marca.

McWilliam (2000) mostró el impacto de las comunidades online en construir relaciones duraderas entre las empresas y los consumidores. Estas relaciones fuertes reflejan la unión psicológica hacia la comunidad y la creencia mutua en el otro (Morgan y Hunt, 1994). La confianza puede ser mejorada cuando los miembros aumentan su dependencia de internet para obtener información de los productos que les ayudarán para tomar las decisiones de compra (Shankar et al., 2003). De esta manera, las empresas pueden utilizar las comunidades online, y los beneficios inherentes a las mismas, para generar confianza y consecuentemente compromiso hacia sus marcas (Ulusu, 2010). Además, sus las personas valoran los beneficios placenteros y funcionales de su marca, su confianza en la misma se incrementará. (Carroll y Ahuvia, 2006; Zhou et al. (2012).

El compromiso afectivo se define como "la consecuencia psicológica de un intercambio o transacción, basado en sentimientos como la identificación, lealtad y afiliación (Verhoef et al., 2002). Este deseo psicológico anexo al producto o marca (Bansal et al., 2004) es un vínculo que motiva al consumidor a permanecer en una relación con una organización. Johnson y Chang (2006) señalaron que el compromiso afectivo determina el deseo de un consumidor para continuar en una relación en el futuro.

El compromiso afectivo ha sido considerado como un antecedente potencial del compromiso del consumidor por distintos investigadores (por ejemplo, Sashi, 2012; Bowden, 2009). Cuando un consumidor se siente activamente comprometido con un producto o servicio, éste desarrolla uniones de comportamiento y emociones con la marca (Guevremont y Grohmann, 2013). Además, el compromiso afectivo ha sido incluido en varias definiciones del compromiso del consumidor. Mollen y Wilson (2010) destacan que el conocimiento y el compromiso afectivo del consumidor es útil para mantener una relación activa con la marca personificada por la web o por otras medidas diseñadas para comunicar el valor de la marca.

Los beneficios percibidos en las redes sociales influyen en la lealtad hacia la marca (Algesheimer et al., 2005). Esta ha sido definida por Peterson y Carrabis (2008) como el nivel de interacción a largo plazo que el consumidor tiene con una marca, sitio web o producto. Estos autores incluyen la lealtad en un índice creado para medir el valor del compromiso del consumidor. De hecho, el compromiso del consumidor se considera como un proceso y no como un estado porque se proyecta sobre una variedad de comportamientos del consumidor y actitudes que dan lugar a consecuencias positivas sobre la marca, como la lealtad (Verhoef et al., 2010; Bowden, 2009; Hollebeek, 2011). En otras palabras, existen estudios que miden el compromiso del consumidor y para ello utilizan otros conceptos aparentemente similares, como la lealtad (Kidd, 2011; Kandampully et al., 2015 y Roberts y Alpert, 2010). Lo cierto es que el compromiso del consumidor incluye varios tipos de comportamientos que dan lugar a la lealtad hacia la marca (Libai, 2011). 
Sánchez Jiménez, M. A.; Fernández Allés, M. T. y Mier-Terán Franco, J. J. Relación entre los beneficios y la obtención de engagement de los usuarios en la comunicación de las redes sociales del sector hotelero

\section{METODOLOGÍA}

En este capítulo se ha establecido la metodología de investigación utilizada para analizar la relación entre las variables presentadas anteriormente. La selección de las páginas de Facebook y Twitter de los hoteles y el planteamiento y la realización del cuestionario han sido tratados en estos dos puntos.

\subsection{Selección de las comunidades online en Facebook y Twitter}

El presente estudio se ha realizado a través de las páginas de Facebook y Twitter de los hoteles o cadena de hoteles de tres a cinco estrellas. Estas dos redes sociales han sido elegidas porque son las más utilizadas por las empresas del sector hotelero (Hmobile, 2015; Beezhotels, 2016). Además, muchas páginas de Facebook y Twitter de los hoteles han ido incorporando varios aspectos atractivos para los usuarios que las siguen (como por ejemplo promociones) con el objetivo de alentar la participación de los miembros y de incidir en otras variables directamente relacionadas con el compromiso del consumidor hacia la marca. Para el estudio se ha considerado el mismo tratamiento si el usuario contesta sobre el Facebook o Twitter del hotel, ya que tal como se ha especificado anteriormente en el estudio de Leung et al. (2013) los resultados obtenidos entre Facebook y Twitter en su utilización como canales de marketing y comunicación son muy similares, lo realmente importante es el uso que se da de esas redes sociales, buscando generar mayores beneficios a través de una mejor experiencia para los usuarios.

\subsection{Planteamiento y realización del cuestionario}

Para la construcción del cuestionario se ha llevado a cabo una búsqueda bibliográfica extensa sobre los ítems que son más adecuados para el estudio, los cuáles han sido aprobados por otros autores. Posteriormente, para validar el cuestionario, se ha realizado una prueba piloto para llegar a la batería definitiva de preguntas y determinar así cuáles son los mejores ítems para cada constructo.

El cuestionario fue dividido en tres secciones: (1) Información sobre la relación del usuario con las redes sociales de los hoteles y del propio hotel elegido e información demográfica (2) Beneficios funcionales, socio-psicológicos, hedónicos y monetarios; y (3) Participación en la comunidad, confianza en la marca, compromiso afectivo y lealtad hacia la marca. Antes de comenzar la primera parte de la encuesta, se realizó una breve introducción del estudio, presentando al investigador, determinando brevemente el tema a tratar, destacando la importancia de la opinión de los encuestados e incidiendo que los datos serían tratados sólo de forma anónima y con fines de investigación.

A continuación, en la Tabla 2 se muestran las variables a analizar y los ítems a medir.

Tabla 2. Variables e ítems de la investigación cuantitativa.

\begin{tabular}{|c|c|c|}
\hline Variables & Ítems de medición & Referencia \\
\hline Beneficios funcionales & $\bullet$ Obtener información actualizada sobre el hotel & De Vries y Carlson \\
\hline
\end{tabular}


Sánchez Jiménez, M. A.; Fernández Allés, M. T. y Mier-Terán Franco, J. J. Relación entre los beneficios y la obtención de engagement de los usuarios en la comunicación de las redes sociales del sector hotelero

\begin{tabular}{|c|c|c|}
\hline & $\begin{array}{l}\text { - Obtener un contenido atractivo } \\
\text { - Poder interactuar directamente con el hotel } \\
\text { - Ver imágenes o videos del hotel }\end{array}$ & $\begin{array}{c}\text { (2014); Kang } \\
\text { (2011); Wang y } \\
\text { Fesenmaier (2004) }\end{array}$ \\
\hline $\begin{array}{l}\text { Beneficios socio- } \\
\text { psicológicos }\end{array}$ & $\begin{array}{l}\text { - Poder compartir tus propias experiencias u } \\
\text { opiniones con otros miembros de la comunidad } \\
\text { - Conocer las experiencias u opiniones de otros } \\
\text { miembros de la comunidad } \\
\text { - Establecer y mantener relaciones con otros } \\
\text { miembros de la comunidad } \\
\text { - Crear un sentido de afiliación o pertenencia con } \\
\text { la comunidad } \\
\text { - Confiar más en el hotel }\end{array}$ & $\begin{array}{c}\text { Kang (2011); } \\
\text { Wang y } \\
\text { Fesenmaier (2004) }\end{array}$ \\
\hline Beneficios hedónicos & $\begin{array}{l}\text { - Entretenerse en la página de Facebook/Twitter } \\
\text { del hotel } \\
\text { - Disfrutar en la página de Facebook/Twitter del } \\
\text { hotel } \\
\text { - Divertirse con otros miembros de la comunidad }\end{array}$ & $\begin{array}{c}\text { Kang (2011); } \\
\text { Wang y } \\
\text { Fesenmaier (2004) }\end{array}$ \\
\hline Beneficios monetarios & $\begin{array}{l}\text { - Obtener descuentos u ofertas especiales } \\
\text { - Obtener mejores precios } \\
\text { - Recibir cupones } \\
\text { - Participar en concursos }\end{array}$ & $\begin{array}{l}\text { Gummerus et al. } \\
\text { (2012); Gwinner et } \\
\text { al. (1998); Lee et } \\
\text { al. (2008) }\end{array}$ \\
\hline $\begin{array}{l}\text { Participación en la } \\
\text { comunidad }\end{array}$ & $\begin{array}{l}\text { - Suelo interesarme en el contenido de la página } \\
\text { de Facebook/Twitter del hotel } \\
\text { - Suelo dar a "me gusta" al contenido de la } \\
\text { página de Facebook/Twitter del hotel } \\
\text { - Suelo compartir el contenido de la página de } \\
\text { Facebook/Twitter del hotel } \\
\text { - Suelo escribir mensajes y respuestas en la } \\
\text { página de Facebook/Twitter del hotel } \\
\text { - Suelo interacciones con otros miembros de la } \\
\text { comunidad } \\
\text { - Suministro información útil al resto de } \\
\text { miembros de la comunidad } \\
\text { - Suelo participar en las actividades que se } \\
\text { ofrecen en la página de Facebook/Twitter del } \\
\text { hotel }\end{array}$ & $\begin{array}{l}\text { Kang (2011); Koh } \\
\text { and Kim (2004); } \\
\text { Gummerus et al. } \\
\text { (2012); Ho (2014) }\end{array}$ \\
\hline Confianza en la marca & $\begin{array}{l}\text { - El hotel es sincero en la información que ofrece } \\
\text { - Se lo que puedo esperar del hotel } \\
\text { - Confío en la marca del hotel }\end{array}$ & $\begin{array}{c}\text { Kang (2011); } \\
\text { Chiang y Jang } \\
\text { (2007); Wilkins et } \\
\text { al. (2009) }\end{array}$ \\
\hline Compromiso afectivo & $\begin{array}{l}\text { - Tengo apego emocional hacia el hotel } \\
\text { - Tengo un fuerte compromiso en mi relación } \\
\text { con el hotel } \\
\text { - Me identifico con el hotel } \\
\text { - El hotel entiende mis necesidades }\end{array}$ & $\begin{array}{l}\text { Harrison-Walker } \\
\text { (2001); Mattila } \\
\text { (2006); Ahluwalia } \\
\text { (2000) }\end{array}$ \\
\hline Lealtad hacia la marca & $\begin{array}{l}\text { - Me considero leal al hotel } \\
\text { - Recomendaría el hotel a otras personas } \\
\text { - El hotel sería mi primera opción de compra en } \\
\text { esa zona } \\
\text { - Hablo de manera positiva sobre el hotel a otras } \\
\text { personas } \\
\text { - Estoy dispuesto a pagar más por este hotel que } \\
\text { por otros por los beneficios que recibo }\end{array}$ & $\begin{array}{l}\text { Zeithaml et al. } \\
\text { (1996); Cambra- } \\
\text { Fierro et al. } \\
\text { (2013); Karjaluoto, } \\
\text { et al. (2014) }\end{array}$ \\
\hline
\end{tabular}

Fuente: Elaboración propia. 
Sánchez Jiménez, M. A.; Fernández Allés, M. T. y Mier-Terán Franco, J. J. Relación entre los beneficios y la obtención de engagement de los usuarios en la comunicación de las redes sociales del sector hotelero

\subsection{Diseño muestral}

Los participantes se seleccionaron por método no probabilístico de sujetos voluntarios. La población para el presente estudio está formada por los fans o seguidores de las páginas de Facebook o Twitter de hoteles. Para el desarrollo de esta investigación se solicitó mediante correo electrónico y teléfono colaboración a hoteles de tres o cinco estrellas que tuvieran presencia en Facebook o Twitter con al menos 300 seguidores, para que añadieran el enlace de la encuesta online en su página de red social. Una vez contada con la aprobación de aquellos hoteles que aceptaron colaborar, estos añadieron el enlace de la encuesta online en su Facebook o Twitter para que estuviera disponible para los usuarios.

La encuesta online al estar a disposición de las redes sociales de los hoteles y al ser dirigida a seguidores se asegura que esta sea respondida por aquellos usuarios que han estado presentes en dicha página del hotel y han comprobado su contenido. Esto es, se ha distribuido a usuarios tanto hombres como mujeres, de al menos 18 años de edad.

Por lo tanto, las condiciones para participar en el estudio son: tener una cuenta de Facebook o Twitter, y a su vez, seguir por Facebook o Twitter a alguna empresa del sector hotelero. Como la encuesta ha sido publicada por los propios hoteles, se facilita que estas condiciones se cumplan en todos aquellos que participen.

En la Tabla 3 se especifica la ficha técnica de este estudio cuantitativo.

Tabla 3. Ficha técnica del estudio cuantitativo.

\begin{tabular}{|c|l|}
\hline Universo & $\begin{array}{l}\text { La población para el presente estudio está formada por los fans o seguidores de las } \\
\text { páginas de Facebook o Twitter de hoteles }=>3.000 .000 \text { (anexo IV) }\end{array}$ \\
\hline Muestra & 164 encuestas válidas \\
\hline $\begin{array}{c}\text { Nivel de } \\
\text { confianza }\end{array}$ & $\mathrm{a}=95,5 \%$ \\
\hline $\begin{array}{c}\text { Error de } \\
\text { muestreo }\end{array}$ & $7,8 \%$ (utilizando el supuesto de máxima indeterminación $\mathrm{p}=\mathrm{q}=50 \%$ ) \\
\hline $\begin{array}{c}\text { Tipo de } \\
\text { muestreo }\end{array}$ & Por método no probabilístico de sujetos voluntarios. \\
\hline $\begin{array}{c}\text { Trabajo de } \\
\text { campo }\end{array}$ & Realizado en octubre-noviembre de 2017 \\
\hline
\end{tabular}

Fuente: Elaboración propia.

\section{RESULTADOS}

Se han obtenido en total 164 respuestas al cuestionario, todos ellos seguidores de una página de Facebook o Twitter de un hotel o cadena de hoteles. Para el contraste de hipótesis se empleó la técnica de regresión lineal múltiple. Tal como explica Moore (2009) la regresión es un modelo matemático que permite predecir el valor de 
Sánchez Jiménez, M. A.; Fernández Allés, M. T. y Mier-Terán Franco, J. J. Relación entre los beneficios y la obtención de engagement de los usuarios en la comunicación de las redes

una variable dependiente $(Y)$ en función de los valores de la variable independiente $(X)$. Dicho de otra forma, los valores de $Y$ son función de los valores de $X$.

A continuación, se especifican los resultados para cada una de las relaciones mediante la interpretación los coeficientes de regresión.

\subsection{Relación entre los beneficios y la participación}

$Y=$ participación

$\mathrm{X} 1=$ Beneficios funcionales, $\mathrm{X} 2=$ Beneficios socio-psicológicos, $\mathrm{X} 3=$ Beneficios hedónicos, $\mathrm{X} 4=$ Beneficios monetarios

Los datos de la Tabla 4 confirman lo que se había detectado en el análisis de correlaciones, la variable "beneficios monetarios" no se asocia a la participación $(p=0,242)$. Se descubre también que la variable "beneficios funcionales", que antes nos había revelado una correlación baja pero significativa con participación, ahora nos muestra ausencia de relación con esta variable dependiente $(p=0,436)$. Por tanto, sólo tienen una contribución significativa a la explicación de la participación las variables "beneficios socio-psicológicos" y "beneficios hedónicos", siendo esta última la con mayor peso en la explicación de la variabilidad de la variable dependiente "participación" (Beta B. Hedónicos=0,516). Concretamente, en este modelo, un aumento en un punto en la escala de beneficios hedónicos produce un aumento de 1,3341 puntos en la variable "participación", cuando todos los otros tipos de beneficios son iguales a 0 , es decir están controlados. Por otro lado, un aumento de un punto en los beneficios socio-psicológicos producirá un aumento de 0,510 puntos en la variable "participación", manteniendo los otros factores controlados.

Tabla 4. Coeficientesa (participación y beneficios funcionales, socio-psicológicos, hedónicos y monetarios).

\begin{tabular}{|c|c|c|c|c|c|c|c|c|}
\hline & \multirow[t]{2}{*}{ Modelo } & \multicolumn{2}{|c|}{$\begin{array}{l}\text { Coeficientes no } \\
\text { estandarizados }\end{array}$} & \multirow{3}{*}{$\begin{array}{c}\text { Coeficientes } \\
\text { tipificados } \\
\text { Beta } \\
\end{array}$} & \multirow[t]{2}{*}{$\mathbf{t}$} & \multirow[t]{2}{*}{ Sig. } & \multicolumn{2}{|c|}{$\begin{array}{l}\text { Estadísticos de } \\
\text { colinealidad }\end{array}$} \\
\hline & & B & Error típ. & & & & Tolerancia & FIV \\
\hline \multirow{5}{*}{1} & (Constante) & $-4,855$ & 5,709 & &,- 850 & ,397 & & \\
\hline & $\begin{array}{l}\text { Beneficios } \\
\text { Funcionales }\end{array}$ & 204 & 262 & ,056 & ,781 & ,436 & 716 & 1,396 \\
\hline & $\begin{array}{c}\text { Beneficios socio- } \\
\text { psicológicos }\end{array}$ & ,510 & , 154 & ,262 & 3,317 & ,001 & ,596 & 1,677 \\
\hline & $\begin{array}{l}\text { Beneficios } \\
\text { Hedónicos }\end{array}$ & 1,344 & , 185 & ,516 & 7,283 & ,000 & ,741 & 1,350 \\
\hline & $\begin{array}{l}\text { Beneficios } \\
\text { Monetarios }\end{array}$ &,- 185 & , 158 &,- 082 & $-1,176$ & ,242 & 767 & 1,304 \\
\hline
\end{tabular}

Fuente: elaboración propia.

\subsection{Relación entre los beneficios y la confianza hacia el hotel}

$Y=$ Confianza

Revista de Comunicación de la SEECI. 15 marzo 2019 /15 julio, 2019, nº 48, 125-148 
Sánchez Jiménez, M. A.; Fernández Allés, M. T. y Mier-Terán Franco, J. J. Relación entre los beneficios y la obtención de engagement de los usuarios en la comunicación de las redes

$X 1=$ Beneficios funcionales, $X 2=$ Beneficios socio-psicológicos, $X 3=$ Beneficios hedónicos, X4=Beneficios monetarios

En la Tabla 5 se verifica que las variables "beneficios funcionales", "sociopsicológicos" y "monetarios" tienen una contribución significativa en la explicación de la variabilidad de la confianza. Los beneficios monetarios son los que tienen una mayor influencia en la confianza (beta $=0,375$ ), mientras los beneficios sociopsicológicos tienen una contribución menor (beta $=0,170$ ), pero significativa. Los beneficios hedónicos, en cambio, no alcanzan el nivel de significación $(p=0,078)$, por lo que su impacto no es relevante en la explicación de la variabilidad de la confianza.

Concretamente, en este modelo, un aumento en un punto en la escala de beneficios monetarios produce un aumento de 0,260 puntos en la variable "confianza", cuando todos los otros tipos de beneficios son iguales a 0, es decir están controlados. Por otro lado, un aumento de un punto en los beneficios funcionales producirá un aumento de 0,220 puntos en la variable "confianza", mientras que un aumento de un punto en los beneficios socio-psicológicos sólo produce un aumento de 0,100 puntos en la confianza, manteniendo en ambos casos los otros factores controlados.

Tabla 5. Coeficientesa (confianza y beneficios funcionales, socio-psicológicos, hedónicos y monetarios).

\begin{tabular}{|c|c|c|c|c|c|c|c|c|c|}
\hline \multirow{2}{*}{ Modelo } & \multicolumn{2}{|c|}{$\begin{array}{l}\text { Coeficientes no } \\
\text { estandarizados }\end{array}$} & \multirow{2}{*}{$\begin{array}{c}\text { Coeficientes } \\
\text { tipificados } \\
\text { Beta }\end{array}$} & \multirow[t]{2}{*}{$\mathbf{t}$} & \multirow{2}{*}{ Sig. } & \multicolumn{2}{|c|}{$\begin{array}{c}\text { Intervalo de } \\
\text { confianza de } \\
95,0 \% \text { para B }\end{array}$} & \multicolumn{2}{|c|}{$\begin{array}{l}\text { Estadísticos de } \\
\text { colinealidad }\end{array}$} \\
\hline & B & Error típ. & & & & \begin{tabular}{|l} 
Límite \\
inferior
\end{tabular} & \begin{tabular}{|c|} 
Límite \\
superior
\end{tabular} & Tolerancia & FIV \\
\hline (Constante) & 1,397 & 1,786 & & ,782 &, 435 & $-2,133$ & 4,927 & & \\
\hline $\begin{array}{l}\text { Beneficios } \\
\text { socio- } \\
\text { psicológicos }\end{array}$ & 100 & ,047 & 170 & 2,148 & ,033 & ,008 & 192 & 633 & 1,579 \\
\hline $\begin{array}{l}\text { Beneficios } \\
\text { Hedónicos }\end{array}$ & 103 & ,058 & 129 & 1,774 & ,078 &,- 012 & 218 & ,745 & 1,342 \\
\hline $\begin{array}{l}\text { Beneficios } \\
\text { Monetarios }\end{array}$ & 260 & ,050 & 375 & 5,221 & ,000 & , 162 & 359 & 767 & 1,303 \\
\hline $\begin{array}{c}\text { Beneficios } \\
\text { Funcionales }\end{array}$ & 220 & ,081 & 201 & 2,723 & ,007 & ,060 & 380 & ,726 & 1,377 \\
\hline
\end{tabular}

Fuente: elaboración propia.

\subsection{Relación entre los beneficios y el compromiso afectivo}

$Y=$ Compromiso afectivo

$X 1=$ Beneficios funcionales, $X 2=$ Beneficios socio-psicológicos, $X 3=$ Beneficios hedónicos, $\mathrm{X} 4=$ Beneficios monetarios 
Sánchez Jiménez, M. A.; Fernández Allés, M. T. y Mier-Terán Franco, J. J. Relación entre los beneficios y la obtención de engagement de los usuarios en la comunicación de las redes sociales del sector hotelero

En la Tabla 6 se verifica que sólo tienen una contribución significativa a la explicación de la variable "compromiso afectivo" los beneficios socio-psicológicos y los beneficios hedónicos.

Los beneficios hedónicos son los que tienen una mayor influencia en el compromiso afectivo (beta $=0,289$ ) y le siguen los beneficios socio-psicológicos (beta $=0,255)$. Los beneficios monetarios $(p=0,202)$ y los funcionales $(p=0,223)$, en cambio, no alcanzan el nivel de significación, por lo que su impacto no es relevante en la explicación de la variabilidad del compromiso afectivo.

Concretamente, en este modelo, un aumento en un punto en la escala de beneficios hedónicos produce un aumento de 0, 371 puntos en la variable "compromiso afectivo", cuando todos los otros tipos de beneficios son iguales a 0, es decir están controlados. Por otro lado, un aumento de un punto en los beneficios socio-psicológicos producirá un aumento de 0,243 puntos en esta variable dependiente, manteniendo en ambos casos los otros factores controlados.

Tabla 6. Coeficientes (compromiso afectivo y beneficios funcionales, sociopsicológicos, hedónicos y monetarios).

\begin{tabular}{|c|c|c|c|c|c|c|c|c|}
\hline \multirow{2}{*}{\multicolumn{2}{|c|}{ Modelo }} & \multicolumn{2}{|c|}{$\begin{array}{l}\text { Coeficientes no } \\
\text { estandarizados }\end{array}$} & \multirow{2}{*}{\begin{tabular}{|c|}
$\begin{array}{c}\text { Coeficientes } \\
\text { tipificados }\end{array}$ \\
Beta \\
\end{tabular}} & \multirow[t]{2}{*}{$\mathbf{t}$} & \multirow[t]{2}{*}{ Sig. } & \multicolumn{2}{|c|}{$\begin{array}{c}\text { Estadísticos de } \\
\text { colinealidad }\end{array}$} \\
\hline & & B & Error típ. & & & & Tolerancia & FIV \\
\hline \multirow{5}{*}{1} & (Constante) & ,240 & 3,349 & & ,072 & ,943 & & \\
\hline & $\begin{array}{c}\text { Beneficios } \\
\text { Funcionales }\end{array}$ & 185 & 151 & 105 & 1,223 & 223 & 727 & 1,375 \\
\hline & $\begin{array}{l}\text { Beneficios socio- } \\
\text { psicológicos }\end{array}$ & 243 & ,087 & 255 & 2,787 & ,006 & ,634 & 1,576 \\
\hline & $\begin{array}{l}\text { Beneficios } \\
\text { Hedónicos }\end{array}$ & ,371 & 109 & 289 & 3,418 & ,001 & 745 & 1,342 \\
\hline & $\begin{array}{l}\text { Beneficios } \\
\text { Monetarios }\end{array}$ &,- 120 & ,094 &,- 106 & $\begin{array}{c}- \\
1,281\end{array}$ & 202 & 770 & 1,299 \\
\hline
\end{tabular}

Fuente: Elaboración propia.

\subsection{Relación entre los beneficios y la lealtad hacia el hotel}

$Y=$ Lealtad

$\mathrm{X} 1=$ Beneficios funcionales, $\mathrm{X} 2=$ Beneficios socio-psicológicos, $\mathrm{X} 3=$ Beneficios hedónicos, X4=Beneficios monetarios

En la Tabla 7 se puede comprobar que las variables "beneficios funcionales", "socio-psicológicos" y "monetarios" tienen una contribución significativa en la explicación de la variabilidad de la lealtad. Los beneficios monetarios son los que tienen una mayor influencia en la lealtad (beta $=0,375$ ), mientras los beneficios sociopsicológicos tienen una contribución menor (beta $=0,170$ ), pero significativa. Los beneficios hedónicos, en cambio, no alcanzan el nivel de significación $(p=0,078)$, por lo que su impacto no es relevante en la explicación de la variabilidad de la lealtad. 
Sánchez Jiménez, M. A.; Fernández Allés, M. T. y Mier-Terán Franco, J. J. Relación entre los beneficios y la obtención de engagement de los usuarios en la comunicación de las redes sociales del sector hotelero

Concretamente, en este modelo, un aumento en un punto en la escala de beneficios monetarios produce un aumento de 0, 260 puntos en la variable "lealtad", cuando todos los otros tipos de beneficios son iguales a 0 , es decir están controlados. Por otro lado, un aumento de un punto en los beneficios funcionales producirá un aumento de 0,220 puntos en la variable "lealtad", mientras que un aumento de un punto en los beneficios socio-psicológicos sólo produce un aumento de 0,100 puntos en la lealtad, manteniendo en ambos casos los otros factores controlados.

Tabla 7. Coeficientesa (lealtad y beneficios funcionales, socio-psicológicos, hedónicos y monetarios).

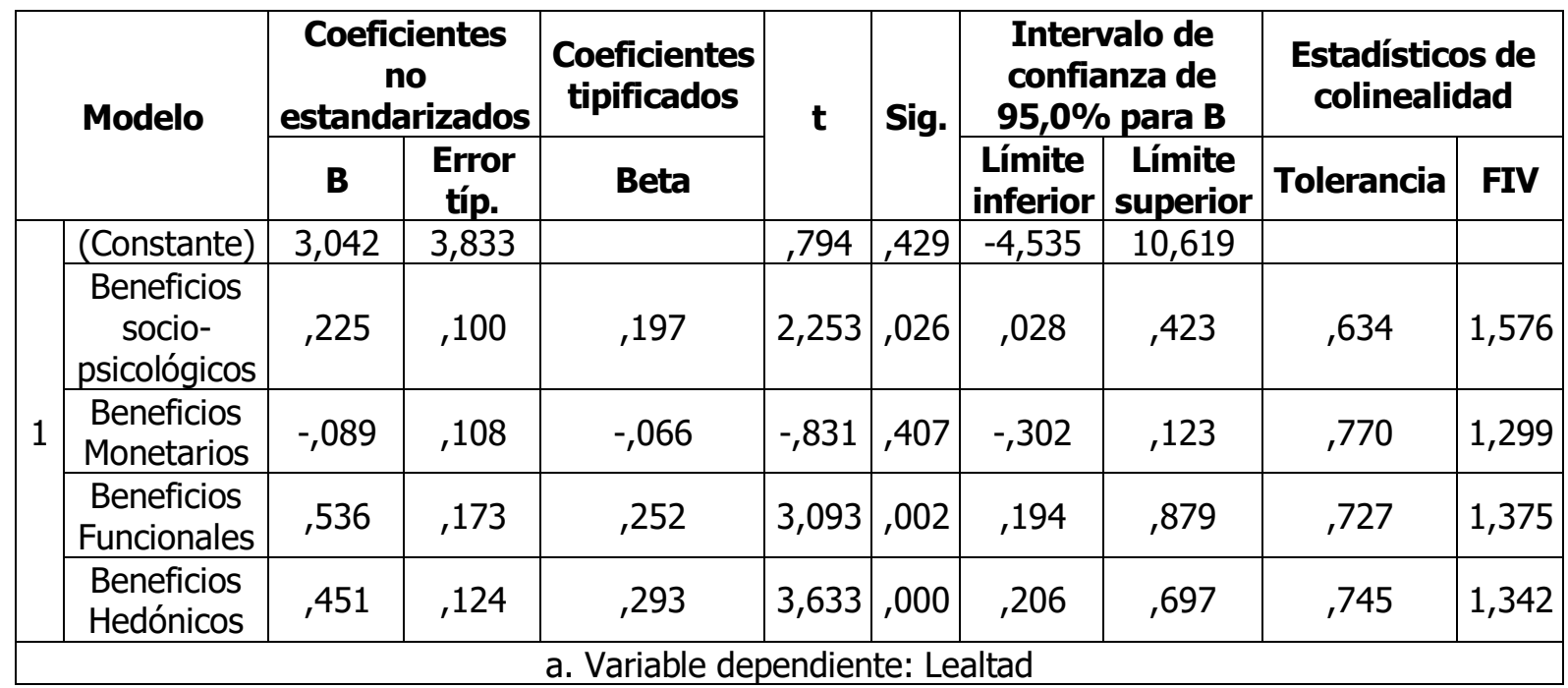

Fuente: elaboración propia.

\section{CONCLUSIONES}

En este apartado se relacionan a modo de resumen en la Tabla 8 los resultados derivados del contraste de las hipótesis planteadas en el estudio, ya comentadas en el punto anterior.

Tabla 8. Contraste de hipótesis en los modelos de regresión múltiple.

\begin{tabular}{|c|c|c|c|c|c|c|}
\hline Hip. & VD & VI & B & Beta & P & Decisión \\
\hline 1 & & & & & & $\begin{array}{c}\text { Contrastada } \\
\text { parcialmente }\end{array}$ \\
\hline & PART & BFUN & 0,204 & 0,056 & 0,436 & No significativa \\
\hline & & BSOC & 0,510 & 0,262 & 0,001 & Significativa \\
\hline & & BHED & 1,344 & 0,516 & $<0,001$ & Significativa \\
\hline & & BMON & $-0,185$ & $-0,082$ & 0,242 & No significativa \\
\hline & & & & & & \\
\hline 2 & & & & & & $\begin{array}{c}\text { Contrastada } \\
\text { parcialmente }\end{array}$ \\
\hline & CONF & BFUN & 0,220 & 0,201 & 0,007 & Significativa \\
\hline & & BSOC & 0,100 & 0,170 & 0,033 & Significativa \\
\hline
\end{tabular}


Sánchez Jiménez, M. A.; Fernández Allés, M. T. y Mier-Terán Franco, J. J. Relación entre los beneficios y la obtención de engagement de los usuarios en la comunicación de las redes sociales del sector hotelero

\begin{tabular}{|c|c|c|c|c|c|c|}
\hline & & BHED & 0,103 & 0,129 & 0,078 & No significativa \\
\hline & & BMON & 0,260 & 0,375 & $<0,001$ & Significativa \\
\hline 3 & & & & & & $\begin{array}{l}\text { Contrastada } \\
\text { parcialmente }\end{array}$ \\
\hline & COMPROM & BFUN & 0,185 & 0,105 & 0,223 & No significativa \\
\hline & & BSOC & 0,243 & 0,255 & 0,006 & Significativa \\
\hline & & BHED & 0,371 & 0,289 & 0,001 & Significativa \\
\hline & & BMON & $-0,120$ & $-0,106$ & 0,202 & No significativa \\
\hline 4 & & & & & & $\begin{array}{l}\text { Contrastada } \\
\text { parcialmente }\end{array}$ \\
\hline & LEALTAD & BFUN & 0,536 & 0,252 & 0,002 & Significativa \\
\hline & & BSOC & 0,225 & 0,197 & 0,026 & Significativa \\
\hline & & BHED & 0,451 & 0,293 & $<0,001$ & Significativa \\
\hline & & BMON & $-0,089$ & $-0,066$ & 0,407 & No significativa \\
\hline
\end{tabular}

Fuente: elaboración propia.

De los cuatro beneficios analizados, únicamente los socio-psicológicos y hedónicos tiene relación con la participación del consumidor, por lo tanto, la participación del usuario en la red social se incrementará mientras mayor conexión tenga con los demás usuarios y mayor entretenimiento perciba en dicha red social. Respecto a la relación entre los beneficios y la confianza hacia el hotel, se comprueba que tanto los beneficios funcionales, socio-psicológicos y monetarios influyen positivamente en la confianza, quedando únicamente excluido el beneficio hedónico. La variable de compromiso afectivo, entendido esta como la vinculación emocional hacia el hotel, solamente tiene una relación positiva con los beneficios sociales y hedónico, quedando excluidos los beneficios funcionales y monetarios, los cuáles no se relacionan a la hora de conseguir un mayor vínculo emocional con el hotel. Por último, una mayor percepción de los beneficios funcionales, sociales y hedónicos va a influir positivamente en la lealtad hacia el hotel, quedando únicamente excluido el interés del usuario a conseguir ofertas económicas mediante el beneficio monetario. Así, cabe destacar la escasa trascendencia del beneficio monetario para conseguir un mayor compromiso con los usuarios.

Por lo tanto, el estudio determina la implicación y las utilidades que obtienen los usuarios en las redes sociales de los hoteles. Además, pone de manifiesto la obtención de beneficios por parte de los usuarios en su intervención en las redes sociales, así como la de un mayor grado de compromiso. De esta manera, se resalta la importancia de ofrecer por parte de los hoteles un contenido atractivo y orientado a los usuarios a través de las redes sociales.

\section{REFERENCIAS}

Ahluwalia, R. (2000). Examination of psychological processes underlying resistance to persuasion. Journal of Consumer Research, 272), 217-232. 
Sánchez Jiménez, M. A.; Fernández Allés, M. T. y Mier-Terán Franco, J. J. Relación entre los beneficios y la obtención de engagement de los usuarios en la comunicación de las redes sociales del sector hotelero

Ahuja, M. K., \& Galvin, J. E. (2003). Socialization in virtual groups. Journal of Management, 29(2), 161-185.

Al-Msallam, S., \& Alhaddad, A. (2016). The effects of social media Marketing in the hotel industry: conceptual model for development of an effective online community. International Journal of Business and Management, 5.

Algesheimer, R.; Dholakia, U. M., \& Herrmann, A. (2005). The social influence of brand community: Evidence from European car clubs. Journal of marketing, 69(3), 19-34.

Bambauer-Sachse, S., \& Mangold, S. (2011). Brand equity dilution through negative online word-of-mouth communication. Journal of Retailing and Consumer Services, 18(1), 38-45.

Bansal, H. S.; Irving, P. G., \& Taylor, S. F. (2004). A three-component model of customer to service providers. Journal of the Academy of marketing Science, 32(3), 234-250.

Becerra, E., \& Badrinarayanan, V. (2013). The influence of brand trust and brand identification on brand evangelism. Journal of Product \& Brand Management, 22(5-6), 371-383.

Beezhotels (2016). Top 5 de redes sociales para hoteles. Recuperado de: http://www.beezhotels.com/blog/top-5-de-redes-sociales-para-hoteles

Bowden, J. (2009). Customer engagement: a framework for assessing customerbrand relationships: the case of the restaurant industry. Journal of Hospitality Marketing \& Management, 18(6), 574-596.

Bressler, S. E., \& Grantham, C. (2000). Communities of commerce: Building internet business communities to accelerate growth, minimize risk, and increase customer loyalty. McGraw-Hill Professional.

Brodie Roderick, J., \& Linda D. Hollebeek (2011). Advancing and Consolidating Knowledge About Customer Engagement. Journal of Service Research, 14(3), 283284.

Buhalis, D. (1998). Strategic uses of information technologies in the tourism industry. Tourism Management, 19(5), 409-421.

Cambra-Fierro, J. J.; Melero-Polo, I., \& Vázquez-Carrasco, R. (2013). Customer engagement. Innovation in non-technical marketing processes. Innovation, 15(3), 326-336.

Carroll, B. A., \& Ahuvia, A. C. (2006). Some antecedents and outcomes of brand love. Marketing letters, 1入2), 79-89. 
Sánchez Jiménez, M. A.; Fernández Allés, M. T. y Mier-Terán Franco, J. J. Relación entre los beneficios y la obtención de engagement de los usuarios en la comunicación de las redes sociales del sector hotelero

Chen, R. (2013). Member use of social networking sites-an empirical examination. Decision Support Systems, 54(3), 1219-1227.

Chiang, C. F., \& Jang, S. S. (2007). The effects of perceived price and brand image on value and purchase intention: Leisure travelers' attitudes toward online hotel booking. Journal of Hospitality \& Leisure Marketing, 15(3), 49-69.

Cruz, B., \& Mendelsohn, J. (2010). Why social media matters to your business. Recuperado de: http://www.cmbinfo.com/cmb-cms/wpcontent/uploads/2010/04/Why Social Media Matters 2010.pdf

De Vries, N. J., \& Carlson, J. (2014). Examining the drivers and brand performance implications of customer engagement with brands in the social media environment. Journal of Brand Management, 21(6), 495-515.

Dholakia, U. M.; Bagozzi, R. P., \& Pearo, L. K. (2004). A social influence model of consumer participation in network-and small-group-based virtual communities. International journal of research in marketing, 21(3), 241-263.

Dowling, G. (2001). Creating Corporate Reputations. Identity, Image, and Performance. Oxford: Oxford University Press.

Furlong, M. S. (1989). An electronic community for older adults: The SeniorNet network. Journal of Communication, 39(3), 145-153.

Gefen, D.; Karahanna, E., \& Straub, D. W. (2003). Trust and TAM in online shopping: an integrated model. MIS quarterly, 2オ(1), 51-90.

Gretzel, U.; Kang, M., \& Lee, W. J. (2008). Differences in Consumer-Generated Media Adoption and Use: A Cross-National Perspective. Journal of Hospitality and Leisure Marketing, 1入1-2), 99-120.

Guevremont, A., \& Grohmann, B. (2013). The impact of brand personality on consumer responses to persuasion attempts. Journal of Brand Management, 20(6), 518-530.

Gummerus, J.; Liljander, V.; Weman, E., \& Pihlström, M. (2012). Customer engagement in a Facebook brand community. Management Research Review, 35(9), 857-877.

Gwinner, K. P.; Gremler, D. D., \& Bitner, M. J. (1998). Relational benefits in services industries: the customer's perspective. Journal of the academy of marketing science, 26(2), 101-114.

Harrison-Walker, L. J. (2001). The measurement of word-of-mouth communication and an investigation of service quality and customer commitment as potential antecedents. Journal of service research, 4(1), 60-75. 
Sánchez Jiménez, M. A.; Fernández Allés, M. T. y Mier-Terán Franco, J. J. Relación entre los beneficios y la obtención de engagement de los usuarios en la comunicación de las redes sociales del sector hotelero

Hays, S.; Page, S. J., \& Buhalis, D. (2013). Social media as a destination marketing tool: its use by national tourism organisations. Current issues in Tourism, 16(3), 211-239.

Hiltz, S. R., \& Wellman, B. (1997). Asynchronous learning networks as a virtual classroom. Communications of the ACM, 40(9), 44-49.

Hmobile (2015). El impacto de las redes sociales en el sector hotelero. Recuperado de http://www.hmobile.es/2015/06/15/el-impacto-de-las-redes-sociales-en-elsector-hotelero/

Ho, C. W. (2014). Consumer behavior on Facebook: does consumer participation bring positive consumer evaluation of the brand? EuroMed Journal of Business, 9(3), 252-267.

Hollebeek, L. (2011). Demystifying customer engagement: exploring the loyalty nexus. Journal of Marketing Management, 277-8), 785-807.

Johnson, R. E., \& Chang, C. H. (2006). It is to continuance as "We" is to affective: the relevance of the self-concept for organizational commitment. Journal of Organizational Behavior, 275), 549-570.

Kandampully, J.; Zhang, T., \& Bilgihan, A. (2015). Customer loyalty: a review and future directions with a special focus on the hospitality industry. International Journal of Contemporary Hospitality Management, 273), 379-414.

Kang, J. (2011). Social media marketing in the hospitality industry: the role of benefits in increasing brand community participation and the impact of participation on consumer trust and commitment toward hotel and restaurant brands. Graduate Theses and Dissertations. 10447. https://lib.dr.iastate.edu/etd/10447

Karjaluoto, H.; Munnukka, J., \& Tikkanen, A. (2014). Are Facebook Brand Community Members Really Loyal to the Brand? 27th Bled eConference eEcosystems, 169-180.

Kidd, J. (2011). Enacting engagement online: framing social media use for the museum. Information Technology \& People, 24(1), 64-77.

Kim, W. G.; Lee, C., \& Hiemstra, S. J. (2004). Effects of an online virtual community on customer loyalty and travel product purchases. Tourism management, 25(3), 343-355.

Koh, J., \& Kim, Y. G. (2004). Knowledge sharing in virtual communities: an ebusiness perspective. Expert systems with applications, 26(2), 155-166.

Langerak, F.; Verhoef, P. C.; Verlegh, P. W., \& de Valck, K. (2003). The effect of members satisfaction with a virtual community on member participation. ERIM 
Sánchez Jiménez, M. A.; Fernández Allés, M. T. y Mier-Terán Franco, J. J. Relación entre los beneficios y la obtención de engagement de los usuarios en la comunicación de las redes sociales del sector hotelero

Report Series Reference No. ERS-2003-004-MKT. Available at SSRN: https://ssrn.com/abstract $=411641$

Lee, Y. K.; Ahn, W. K., \& Kim, K. (2008). A study on the moderating role of alternative attractiveness in the relationship between relational benefits and customer loyalty. International Journal of hospitality \& tourism administration, $9(1)$, 52-70.

Leung, D.; Law, R.; van Hoof, H., \& Buhalis, D. (2013). Social media in tourism and hospitality: A literature review. Journal of Travel \& Tourism marketing, 30(1-2), 3.

Leung, X. Y., Bai, B. y Stahura, K. A. (2015). The marketing effectiveness of social media in the hotel industry: A comparison of Facebook and Twitter. Journal of Hospitality \& Tourism Research, 39(2), 147-169.

Libai, B. (2011). Comment: the perils of focusing on highly engaged customers. Journal of Service Research, 14(3), 275-276.

Litvin, S. W.; Goldsmith, R. E., \& Pan, B. (2008). Electronic word-of-mouth in hospitality and tourism management. Tourism Management, 29(3), 458-468.

Madupu, V. (2006). Online brand community participation: antecedents and consequences, Doctoral dissertation.

Martínez, S. M.; Bernal García, J. J., \& Mellinas, J. P. (2013). Análisis del nivel de presencia de los establecimientos hoteleros de la Región de Murcia en la Web 2.0. Cuadernos de Turismo, 31, 245-261.

Mattila, A. S. (2006). How affective commitment boosts guest loyalty (and promotes frequent-guest programs). Cornell Hotel and Restaurant Administration Quarterly, 47(2), 174-181.

McWilliam, G. (2000). Building stronger brands through online communities. Sloan management review, 41(3), 43.

Mollen, A., \& Wilson, H. (2010). Engagement, telepresence and interactivity in online consumer experience: Reconciling scholastic and managerial perspectives. Journal of business research, 63(9), 919-925.

Moore, D. S. (2005). Estadística aplicada básica. Antoni Bosch editor.

Morgan, R. M., \& Hunt, S. D. (1994). The commitment-trust theory of relationship marketing". The journal of marketing, 20-38.

Naumann, K., \& Lay-Hwa Bowden, J. (2015). Exploring the process of customer engagement, self-brand connections and loyalty. Problems and perspectives in management, 13, 56-66 and 5. 
Sánchez Jiménez, M. A.; Fernández Allés, M. T. y Mier-Terán Franco, J. J. Relación entre los beneficios y la obtención de engagement de los usuarios en la comunicación de las redes sociales del sector hotelero

Peterson, E. T, \& Carrabis, J. (2008). Measuring the immeasurable: Visitor engagement. Web Analytics Demystified, 14, 16.

Potgieter, L. M., \& Naidoo, R. (2017). Factors explaining user loyalty in a social media-based brand community. South African Journal of Information Management, 19(1), 1-9.

Powers, T.; Advincula, D.; Austin, M. S.; Graiko, S., \& Snyder, J. (2012). Digital and social media in the purchase decision process. Journal of advertising research, 52(4), 479-489.

Roberts, C., \& Alpert, F. (2010). Total customer engagement. designing and aligning key strategic elements to achieve growth. Journal of Product \& Brand Management, 19(3), 198-209.

Rosenthal, B., \& Brito, E. P. (2017). How virtual brand community traces may increase fan engagement in brand pages. Business Horizons, 60(3), 375-384.

Sahin, G. G., \& Sengün, G. (2015). The Effects of Social Media on Tourism Marketing: A Study among University Students. Management and Administrative Sciences Review, 4(5), 772-786.

Sashi, C. M. (2012). Customer engagement, buyer-seller relationships, and social media. Management decision, 50(2), 253-272.

Sigala, M. (2007). Web 2.0 in the tourism industry: $A$ new tourism generation and new ebusiness models. Recuperado de http://www.traveldailynews.com/pages/show page/20554

Sirakaya, E., \& A. Woodside (2005). Building and testing theories of decision making by travelers. Tourism Management, 26(6), 815-832.

Song, S., \& Yoo, M. (2016). The role of social media during the pre-purchasing stage. Journal of Hospitality and Tourism Technology, 71 ), 84-99.

Tercero, M. M. (1999). Ciencia y marketing: manual para investigadores y doctorandos en ciencias sociales. Esic Editorial.

Ulusu, Y. (2010). Determinant factors of time spent on facebook: Brand community engagement and usage types. Journal of Yasar University, 18(5), 2949-2957.

Verhoef, P. C.; Franses, P. H., \& Hoekstra, J. C. (2002). The effect of relational constructs on customer referrals and number of services purchased from a multiservice provider: does age of relationship matter? Journal of the Academy of Marketing Science, 30(3), 202-216. 
Sánchez Jiménez, M. A.; Fernández Allés, M. T. y Mier-Terán Franco, J. J. Relación entre los beneficios y la obtención de engagement de los usuarios en la comunicación de las redes sociales del sector hotelero

Verhoef, P. C.; Reinartz, W. J., \& Krafft, M. (2010). Customer engagement as a new perspective in customer management. Journal of Service Research, 13(3), 247252.

Vivek, S.; Beatty, S., \& Morgan, R., (2012). Customer engagement: Exploring customer relationships beyond purchase. Journal of Marketing Theory and Practice, 20(2), 122-146.

Vivek, S.; Beatty, S., \& Morgan, R., (2012). Customer engagement: Exploring customer relationships beyond purchase. Journal of Marketing Theory and Practice, 20(2), 122-146.

Wang, Y., \& Fesenmaier, D. R. (2004). Towards understanding members' general participation in and active contribution to an online travel community. Tourism management, 25(6), 709-722.

Wilkins, H.; Merrilees, B., \& Herington, C. (2009). The determinants of loyalty in hotels. Journal of Hospitality Marketing \& Management, 19(1), 1-21.

Zeithaml, V. A., Berry, L. L. y Parasuraman, A. (1996). "The behavioral consequences of service quality". The Journal of Marketing, 31-46.

Zhou, Z.; Zhang, Q.; Su, C., \& Zhou, N. (2012). How do brand communities generate brand relationships? Intermediate mechanisms". Journal of Business Research, 65(7), 890-895.

\section{AUTORES}

\section{Miguel Ángel Sánchez Jiménez}

Personal docente e Investigador en la Universidad de Cádiz en el departamento de Marketing y Comunicación. Doctor en Marketing y Comunicación con la mención al "Doctorado Europeo" y miembro del Instituto de Investigación para el Desarrollo Social Sostenible (INDESS). Actividad investigadora orientada a las nuevas tecnologías digitales en el marketing y la comunicación, social media, marketing móvil, turismo, etc. Autor de artículos publicados en revistas indexadas y participación en congresos de investigación.

miguelangel.sanchez@uca.es

Orcid ID: https://orcid.org/0000-0002-4271-3522

\section{María Teresa Fernández Allés}

Personal docente e Investigador en la Universidad de Cádiz en el departamento de Marketing y Comunicación. Doctora en Economía. Profesora Titular de Universidad. Investigadora en el ámbito del marketing y el turismo, con especialidad en turismo accesible, marketing turístico, sostenibilidad turística, etc. Docente en el Grado en Administración y Dirección de Empresas y en los Másteres en Dirección de Empresas, Dirección de Empresas Turísticas y Marketing Digital y Social. 
Sánchez Jiménez, M. A.; Fernández Allés, M. T. y Mier-Terán Franco, J. J. Relación entre los beneficios y la obtención de engagement de los usuarios en la comunicación de las redes sociales del sector hotelero

teresa.alles@uca.es

Orcid ID: https://orcid.org/0000-0002-3999-8790

\section{Juan José Mier-Terán Franco}

Personal docente e Investigador en la Universidad de Cádiz en el departamento de Marketing y Comunicación. Profesor Doctor Titular de Universidad, investigador en el ámbito del marketing social y digital, director del Departamento de Marketing y Comunicación de la Universidad de Cádiz, coordinador del Máster de Dirección de Marketing Digital y Social y vicepresidente de la Asociación Internacional de Marketing Público y No Lucrativo.

Juanjose.mier-teran@uca.es

Orcid ID: https://orcid.org/0000-0002-6866-1361 